\title{
Low or Declining Gait Speed is Associated With Risk of Developing Dementia Over 5 Years Among People Aged 85 Years and Over
}

\author{
Jerry Öhlin, Yngve Gustafson, Håkan Littbrand, Birgitta Olofsson, and Annika Toots
}

\begin{abstract}
Improving dementia screening procedures beyond simple assessment of current cognitive performance is timely given the ongoing phenomenon of population aging. A slow or declining gait speed (GS) is a potential early indicator of cognitive decline scarcely investigated in very old people. Here, we investigated the 5-year associations of baseline GS, change in GS, and cognitive function with subsequent dementia development in people aged 85 years and older $(n=296)$ without dementia at baseline. Declining and a slow baseline GS were associated with higher odds of dementia development after adjusting for confounders (e.g., age, sex, and dependency in activities of daily living) and missing GS values at follow-up. The GS decline was associated with cognitive decline in participants who developed dementia. The results support the potential of GS tests to predict future cognitive decline among community- and nursing home-dwelling very old people.
\end{abstract}

Keywords: cognitive decline, dementia disorders, very old people, walking speed

With people living longer in many countries around the world, people aged 80 years and older are now the fastest-growing age group and their numbers are expected to triple by 2050 (United Nations, Department of Economic and Social Affairs, Population Division [UN/DESA], 2015). As populations age, the numbers of people with dementia will also increase substantially (Livingston et al., 2017). Dementia disorders are a leading cause of disability and dependence on others for daily activities among older people, and a challenge to health care providers worldwide (World Health Organization, Alzheimer Disease International [WHO/ADI], 2012). Because there are no curative treatments for dementia, current treatment strategies are focused on management and prevention of symptom progression. Early detection of cognitive impairment may provide persons with dementia and their families time to plan ahead and enable access to interventions and care (Livingston et al., 2017).

Gait speed (GS) has been proposed to be a reliable, valid, and sensitive measure with which to screen older adults for subclinical pathogenetic processes that may compromise overall health and function (Middleton, Fritz, \& Lusardi, 2015). Because gait is dependent on central nervous system function (Morris, Lord, Bunce, Burn, \& Rochester, 2016) in addition to the functions of circulatory, respiratory, and musculoskeletal systems (Studenski et al., 2011), reduced GS can be an early indicator of cognitive impairment. The GS decline has been associated with a heightened risk for dementia in older people by several research groups (Hackett, Davies-Kershaw,

(C) 2021 The Authors. Published by Human Kinetics, Inc. This is an Open Access article distributed under the terms of the Creative Commons Attribution 4.0 International License, CC BY 4.0, which permits unrestricted noncommercial and commercial use, distribution, and reproduction in any medium, provided the original work is properly cited, the new use includes a link to the license, and any changes are indicated. See http://creativecommons.org/licenses/by/4.0. This license does not cover any third-party material that may appear with permission in the article

Öhlin, Gustafson, and Littbrand are with the Department of Community Medicine and Rehabilitation, Geriatric Medicine, Umeå University, Umeå, Sweden. Olofsson is with the Department of Nursing, Umeå University, Umeå, Sweden. Toots is with the Department of Community Medicine and Rehabilitation, Physiotherapy, Umeå University, Umeå, Sweden. Öhlin (jerry.ohlin@umu.se) is corresponding author.
Cadar, Orrell, \& Steptoe, 2018; Montero-Odasso et al., 2018; Welmer, Rizzuto, Qiu, Caracciolo, \& Laukka, 2014). Furthermore, longitudinal studies have shown that GS reduction may precede a dementia diagnosis by up to 9 years (Beauchet et al., 2016; Bullain, Corrada, Perry, \& Kawas, 2016). A recent meta-analysis showed that people over 60-years old with a dual decline in GS and memory were at a higher risk of dementia than those with only a GS or a memory decline (Tian et al., 2020). These results suggest that a low or declining GS may be indicative of future cognitive function, though there is limited information regarding the association of GS in relation to dementia pathogenesis in very old people.

Longitudinal observations of the association between changes in GS and cognition in very old people can be challenging because, in addition to their high risk of mortality, gait deficits become more likely with age, leading to the exclusion of participants who are unable to perform a GS test. Although it is otherwise a reasonable criterion, it cannot be readily generalized to the very old (Peel, Kuys, \& Klein, 2013) given that one in five people aged 85 years and over may be unable to perform a GS test (Öhlin et al., 2020). In the absence of an adequate strategy to deal with missing values, the consequently large volume of missing data may lead to biased results and invalid conclusions (Hayati Rezvan, Lee, \& Simpson, 2015). Moreover, increasing disease burdens with age (Calderón-Larrañaga et al. 2019) may affect detected associations between gait and cognition (Grande et al. 2019).

Given likely changes in the nature of the relationship between GS and dementia with age, studies of this association specifically in very old people are warranted. The primary aim of this study was to investigate the longitudinal association between GS, change in GS $(\Delta \mathrm{GS})$, and dementia development over 5 years in people aged 85 years and over. A secondary aim was to study the association between changes in GS and cognitive function over 5 years within the same population.

\section{Methods}

\section{Setting and Participants}

This study used data from the Umeå 85+/GErontological Regional DAtabase (Umeå85+/GERDA) project, a population-based study of 
the health, and living situation of both community- and nursing home-dwelling older people. Data collection was recurrent (5-year interval) in selected urban and rural municipalities in Västerbotten County, northern Sweden (years 2000/2002, 2005/2007, 2010/2012, and 2015/2017) and in Österbotten County, western Finland (years 2006 and 2011). The last follow-up was in 2017. Participants were invited to participate based on a systematic sampling of individuals from national tax and population registers according to date of birth. From a randomized starting point, every other person turning 85 years old, every person turning 90 years old, and every person turning 95 years old or over that year were invited to participate. Previous participants were invited to participate in subsequent data collections that were repeated every fifth year. All participants were sent a letter detailing the Umeå85+/GERDA study and then invited by phone to participate. A home visit was scheduled for each individual who provided informed consent to participate in the Umeå85+/GERDA study. When cognitive impairment was suspected, the study information was provided to and assent was obtained from a relative. Completion of data collection took approximately a year for each of the four cohorts. The visits were conducted in order of decreasing age of the participants and in participants' homes to accommodate for impaired mobility or remote living. Medical professionals (physicians, nurses, and physiotherapists) with study protocol training conducted structured interviews and assessments, including collecting data on participants' medical history, and prescribed medications, reviewing medical records, and conducting interviews with relatives or care personnel when required. The inclusion criteria for this study were Umeå85+/GERDA project participation, being without dementia at enrollment, completing tests of GS and cognition during a home visit, and completing a cognition test at a home visit 5 years later. As shown in Figure 1, of 1,867 eligible individuals, 296 were included in baseline and follow-up data collection (2000/2002, $n=95 ; 2005 / 2007$ and 2006, $N=114$; and $2010 / 2012, n=87$ ). This study was approved by the regional ethics review board in Umeå (registration numbers: 99-326, 05-063 M, 09178 M, 2018-188-32M) and the ethics committee of Vaasa Central Hospital (registration numbers: 05-87 and 10-54).

\section{Target Variables}

The GS was measured over $2.4 \mathrm{~m}$ ( 8 feet), as a part of the original short physical performance battery (Guralnik et al. 1994). Participants were instructed to start by standing still behind a marking on the floor and walk at a self-paced speed past a second marking. Time was measured on a stopwatch from the moment walking started to the moment the first foot passed the second marking. The test was conducted twice and mean GS was calculated in meter per second. If only one measure was recorded $(n=3)$, it was included in the analysis. Participants who could not perform the GS test at the second home visit $(n=51)$ were included, and the reason for nonperformance was recorded and categorized as cognition, physical impairment, motivation, or other (pain, unknown).

Global cognitive function was measured using the Mini-Mental State Examination (MMSE), a common screening tool for dementia with satisfactory reliability (Tombaugh \& McIntyre, 1992) and validity (Creavin et al. 2016). The MMSE has 11 items related to orientation in time and place, memory, attention, and visuospatial function. Total scores range from 0 to 30 , with a higher score indicating better cognitive function. A minimal score was given on single items if participants refused to answer or could not answer due to impaired vision, hearing, or motor function.
Dementia diagnoses were verified according to the Diagnostic and Statistical Manual of Mental Disorders, fourth edition, Text Revision (American Psychiatric Association [APA], 2000) using information from medical records, prescriptions, and assessments including the MMSE (Folstein, Folstein, \& McHugh, 1975), 15-item Geriatric Depression Scale (Sheikh \& Yesavage, 1986), Philadelphia Geriatric Center Morale Scale (Lawton, 1975; Niklasson et al., 2015), Life Orientation Scale (Tilvis et al., 2012), Barthel activities of daily living (ADL) index (Collin, Wade, Davies, \& Horne, 1988), Organic Brain Syndrome Scale (Jensen, Dehlin, \& Gustafson, 1993), vision tests, and hearing tests. One experienced specialist in geriatric medicine reviewed all medical diagnoses in Sweden and Finland.

\section{Potential Confounders}

Sociodemographic and anthropometric data were collected. Body mass index was calculated. Years of education was dichotomized into $<8$ years or $\geq 8$ years. Systolic and diastolic blood pressure were measured with a calibrated sphygmomanometer and stethoscope after $5 \mathrm{~min}$ of rest in a supine position. Depressive symptoms were measured with the 15-item Geriatric Depression Scale, with higher scores indicating more depressive symptoms (Sheikh \& Yesavage, 1986). For participants with $\leq 5$ missing answers, a total score was imputed by multiplying the mean score of answered questions by 15 . Dependence in personal ADL was measured with the Barthel ADL index (score range, 0-20), with lower scores indicating higher dependence (Collin et al., 1988). Impaired hearing and vision were defined as the inability to hear a conversation at usual speaking volume within $1 \mathrm{~m}$, with or without hearing aid, and to read a word in 5-mm capital letters at reading distance, with or without glasses, respectively.

\section{Statistical Analysis}

Data are reported as mean with $S D$ s and frequencies with percentages of the total sample and according to dementia development. Change in MMSE score ( $\triangle \mathrm{MMSE}$ ) and $\triangle \mathrm{GS}$ were calculated as follow-up minus baseline values. Potential confounders for all adjusted analyses were chosen from preselected baseline variables that were believed to influence both target variables. Variables that associated with target variables $(p<.15)$ in univariate logistic or linear regression analyses were inspected for multicollinearity and excluded if Pearson's $R$ or Spearman's Rho exceeded 0.5.

GS and dementia development. Logistic regression was used to calculate unadjusted and adjusted odds ratios and 95\% confidence intervals (95\% CIs) for $\Delta \mathrm{GS}$ and baseline GS and their respective associations with dementia development. Regression analyses for baseline GS and $\Delta \mathrm{GS}$ were adjusted for age, sex, body mass index, history of malignancy, nursing home residency, and Barthel ADL index score; the baseline GS regression analysis was additionally adjusted for history of hip fracture and baseline MMSE. Use of a walking aid during GS testing was removed from both logistic regressions in favor of Barthel ADL index scores (Pearson's $R=-.514, p<.001)$.

Changes in GS and MMSE score. Linear regression was used to calculate unadjusted and adjusted unstandardized beta $(\beta)$ values with $95 \%$ CIs between $\triangle \mathrm{GS}$ and MMSE score data for the total sample and according to dementia development at follow-up. Baseline GS was removed from all three linear analyses for exceeding a multicollinearity threshold of 0.5 with $\Delta \mathrm{GS}$ (Pearson's $r=-.631, p<.001)$. All three linear regression analyses were 


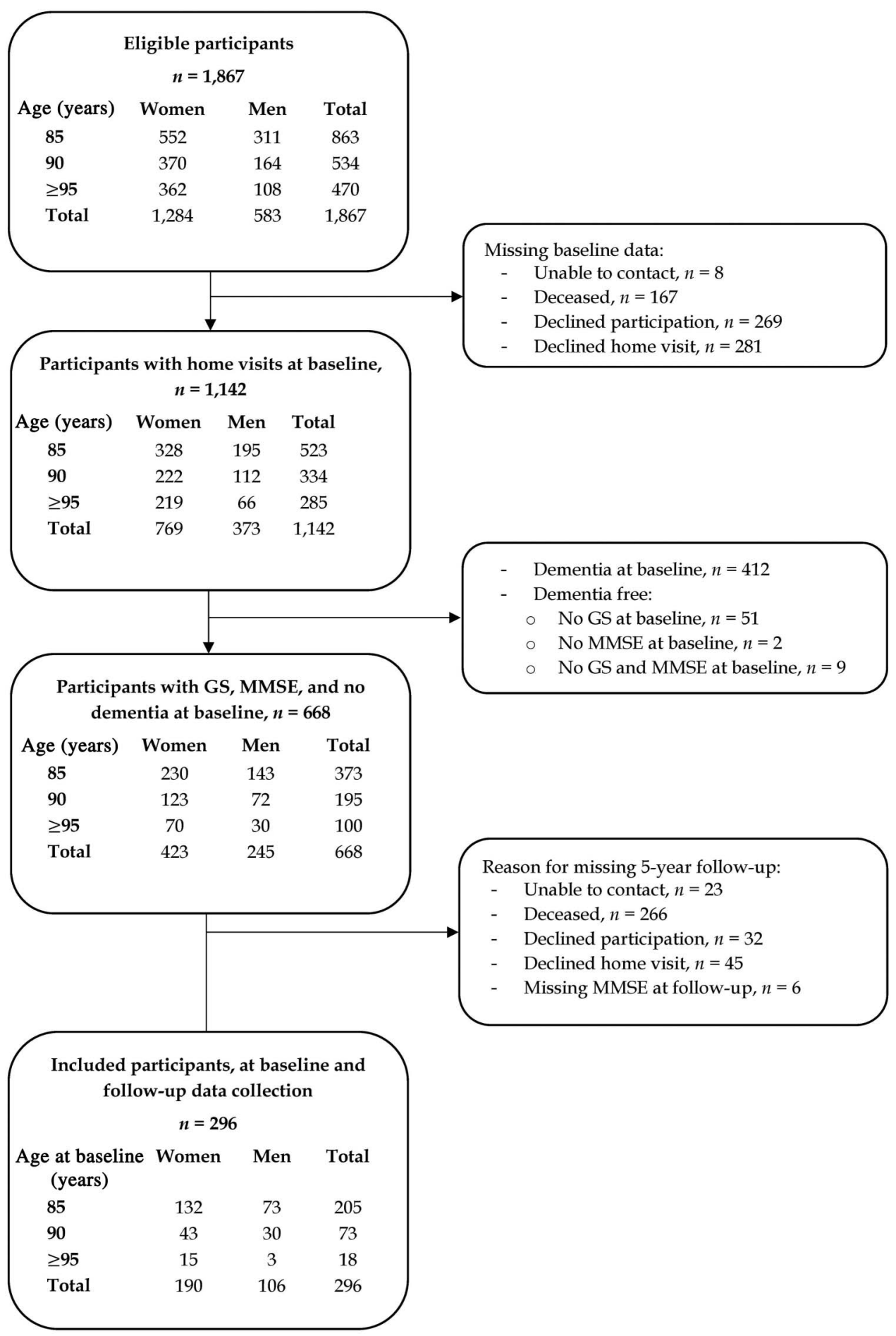

Figure 1 - Flowchart of inclusion procedure. MMSE=Mini-Mental State Examination; GS = gait speed.

adjusted for baseline age, sex, body mass index, history of malignancy, and use of walking aid during GS test.

Missing GS values. Multiple imputation was conducted to estimate missing GS values at follow-up $(n=51)$. The number of generated imputed data sets was set equal to the percentage of missing GS values ( $n=17$; van Buren, 2012). A predefined strategy for selecting predictors was used, selecting baseline GS together with variables at follow-up that were target variables (GS, MMSE score, and dementia development), background characteristics that associated $(r>.3)$ with target variables (age, sex, MMSE score, dementia diagnosis, nursing home resident, Barthel ADL score index, use of walking aid during GS test, analgesics, and antidepressants); and causes for missing GS values (physical impairment $n=34$ ); and other reasons (cognition, $n=1$, motivation, $n=6$, other $n=5$, and reason unknown $n=5$ ). Two restrictions were implemented using the same predictors. Participants with missing GS values due to a physical reason had the maximum imputed value restricted to the lowest observed value $(0.1 \mathrm{~m} / \mathrm{s})$, 
because if they had been able to perform the GS test, it stands to reason their pace would be slow. For participants with other reasons for missing GS values and hence greater uncertainty regarding possible pace, the highest possible imputed GS values were restricted to the maximum observed value $(1.2 \mathrm{~m} / \mathrm{s})$.

Analyses were performed in SPSS Statistics software for Windows (version 24; IBM Corp., Armonk, NY). All statistical tests were two-tailed. The $p$ values $<.05$ were considered statistically significant.

\section{Results}

Demographic and baseline assessment data for the total cohort of 296 participants, for those who later developed dementia (Dem group, $n=98$ ), and for those who did not develop dementia (NoDem group, $n=198$ ), are summarized in Table 1 . Mean baseline, mean follow-up, and change from baseline to 5-year follow-up values for GS and MMSE scores are reported for the total cohort and for the Dem group and NoDem groups in Table 2. About one third of the

Table 1 Background Characteristics of Participants in Total Sample at Baseline, and According to Dementia Development at Baseline and Follow-up

\begin{tabular}{|c|c|c|c|c|c|}
\hline \multirow[b]{2}{*}{ Characteristic } & \multirow{2}{*}{$\begin{array}{l}\text { Whole cohort } \\
\qquad \begin{array}{c}n=296 \\
\text { Baseline }\end{array}\end{array}$} & \multicolumn{2}{|c|}{$\begin{array}{l}\text { Developed dementia } \\
\qquad n=98\end{array}$} & \multicolumn{2}{|c|}{$\begin{array}{l}\text { Did not develop dementia } \\
\qquad n=198\end{array}$} \\
\hline & & Baseline & Follow-up & Baseline & Follow-up \\
\hline Age range (years) & $\begin{array}{c}87.0 \pm 3.2 \\
(84-99)\end{array}$ & $\begin{array}{l}87.7 \pm 3.6 \\
(84-99)\end{array}$ & $\begin{array}{l}92.3 \pm 3.7 \\
(89-104)\end{array}$ & $\begin{array}{c}86.7 \pm 2.9 \\
(84-98)\end{array}$ & $\begin{array}{l}91.4 \pm 3.0 \\
(89-103)\end{array}$ \\
\hline \multicolumn{6}{|l|}{ Age group (years) } \\
\hline $85-89$ & $205(69.3)$ & $59(60.2)$ & $\mathrm{n} / \mathrm{a}$ & $146(73.7)$ & $\mathrm{n} / \mathrm{a}$ \\
\hline $90-94$ & $73(24.7)$ & $30(30.6)$ & $59(60.2)$ & $43(21.7)$ & $146(73.7)$ \\
\hline$\geq 95$ & $18(6.1)$ & $9(9.2)$ & $39(39.8)$ & $9(4.5)$ & $52(26.3)$ \\
\hline Women & $190(64.2)$ & $62(63.3)$ & $62(63.3)$ & $128(64.6)$ & $128(64.6)$ \\
\hline Nursing home resident & $26(8.8)$ & $15(15.3)$ & $57(59.4)$ & $11(5.6)$ & $28(14.5)$ \\
\hline Lives alone & $212(71.6)$ & $75(76.5)$ & $76(82.6)$ & $137(69.2)$ & $153(78.5)$ \\
\hline Education $<8$ years, $n=295$ & $191(64.5)$ & $66(68.0)$ & $66(68.0)$ & $125(63.1)$ & $125(63.1)$ \\
\hline Currently smoking & $12(4.1)$ & $5(5.1)$ & $3(3.1)$ & $7(3.5)$ & 7 (3.6) \\
\hline \multicolumn{6}{|l|}{ Diagnoses and medical conditions } \\
\hline Parkinson disease & $3(1.0)$ & $2(2.0)$ & $3(3.1)$ & $1(0.5)$ & $1(0.5)$ \\
\hline Current depressive disorder & $53(17.9)$ & $20(20.4)$ & $41(41.8)$ & $33(16.7)$ & $47(23.7)$ \\
\hline Cerebrovascular disease & $51(17.2)$ & $14(14.3)$ & $29(29.6)$ & $37(18.7)$ & $44(22.2)$ \\
\hline Myocardial infarction previous year & $2(0.7)$ & $2(2.0)$ & $1(1.0)$ & $0(0)$ & $5(2.5)$ \\
\hline Heart failure & $23(7.8)$ & $10(10.2)$ & $15(15.3)$ & $13(6.6)$ & $27(13.6)$ \\
\hline History of hip fracture & $30(10.1)$ & $16(16.3)$ & $23(25.0)$ & $14(7.1)$ & $20(10.5)$ \\
\hline Diabetes & 35 (11.8) & $10(10.2)$ & $12(12.2)$ & $25(12.6)$ & $32(16.2)$ \\
\hline Osteoarthritis & $139(47.0)$ & $40(40.8)$ & $45(45.9)$ & $99(50.0)$ & $105(53.0)$ \\
\hline History of malignancy & 59 (19.9) & $14(14.3)$ & $23(23.5)$ & $45(22.7)$ & $59(29.8)$ \\
\hline \multicolumn{6}{|l|}{ Routine prescription medications ${ }^{\mathrm{a}}$} \\
\hline Benzodiazepines & 55 (18.6) & $21(21.4)$ & $26(26.8)$ & $34(17.2)$ & $41(20.7)$ \\
\hline$\beta$-blockers & $119(40.2)$ & $35(35.7)$ & $39(40.2)$ & $84(42.2)$ & $96(48.5)$ \\
\hline Antidepressants & $19(6.4)$ & $7(7.1)$ & $22(22.7)$ & $12(6.1)$ & $22(11.1)$ \\
\hline Diuretics & $127(42.9)$ & $43(43.9)$ & $42(43.3)$ & $84(42.4)$ & $101(51.0)$ \\
\hline Analgesics & $74(25.0)$ & $28(28.6)$ & $50(51.5)$ & $46(23.2)$ & $57(28.8)$ \\
\hline Neuroleptics & $13(4.4)$ & $6(6.1)$ & $11(11.3)$ & $7(3.5)$ & $6(3.0)$ \\
\hline Number of prescribed medications & $5.1 \pm 3.4$ & $5.2 \pm 3.6$ & $7.1 \pm 3.8$ & $5.0 \pm 3.3$ & $6.4 \pm 3.6$ \\
\hline \multicolumn{6}{|l|}{ Assessments } \\
\hline BMI (weight/length ${ }^{2}$ ), $n=294$ & $25.9 \pm 4.1$ & $25.3 \pm 3.6$ & $24.0 \pm 4.4$ & $26.2 \pm 4.2$ & $25.4 \pm 4.3$ \\
\hline Systolic blood pressure, $n=292$ & $153.0 \pm 22.1$ & $153.6 \pm 26.7$ & $139.1 \pm 21.3$ & $152.7 \pm 19.5$ & $142.6 \pm 20.1$ \\
\hline Diastolic blood pressure, $n=292$ & $76.3 \pm 10.8$ & $77.7 \pm 11.2$ & $72.4 \pm 13.2$ & $75.7 \pm 10.5$ & $72.9 \pm 12.6$ \\
\hline Barthel ADL Index (0-20) & $19.5 \pm 1.1$ & $19.3 \pm 1.4$ & $13.8 \pm 6.0$ & $19.7 \pm 0.9$ & $18.5 \pm 2.7$ \\
\hline Geriatric Depression Scale (0-15), $n=294$ & $2.9 \pm 2.3$ & $3.2 \pm 2.1$ & $3.9 \pm 3.1$ & $2.8 \pm 2.3$ & $3.2 \pm 2.3$ \\
\hline Vision impairment & $153(51.7)$ & $50(51.0)$ & $54(56.3)$ & $103(52.0)$ & $112(56.9)$ \\
\hline Hearing impairment & $91(30.7)$ & $32(32.7)$ & $47(48.0)$ & $59(29.8)$ & $86(44.3)$ \\
\hline
\end{tabular}

Note. Data presented as mean \pm standard deviation or $N(\%)$. ADL $=$ activities of daily living; $\mathrm{BMI}=$ body mass index.

${ }^{a}$ One participant declined medical chart review and provided no information regarding prescribed medications. 
Table 2 MMSE Scores, Walking Aid Use in GS Testing, and GS and Changes in GS Over 5 Years in the Total Sample at Baseline and According to Dementia Development at Baseline and Follow-Up

\begin{tabular}{|c|c|c|c|c|c|}
\hline \multirow[b]{2}{*}{ Measure } & \multirow{2}{*}{$\begin{array}{c}\begin{array}{c}\text { Total } \\
n=296\end{array} \\
\text { Baseline }\end{array}$} & \multicolumn{2}{|c|}{$\begin{array}{l}\text { Developed dementia } \\
\qquad n=98\end{array}$} & \multicolumn{2}{|c|}{$\begin{array}{l}\text { Did not develop dementia } \\
\qquad n=198\end{array}$} \\
\hline & & Baseline & Follow-up & Baseline & Follow-up \\
\hline MMSE (0-30) & $26.2 \pm 2.7$ & $25.2 \pm 3.0$ & $15.8 \pm 6.3$ & $26.7 \pm 2.3$ & $25.1 \pm 3.4$ \\
\hline MMSE decline & $-4.2 \pm 5.9$ & & $-9.4 \pm 6.4$ & & $-1.6 \pm 1.5$ \\
\hline Baseline GS (m/s observed) & $0.64 \pm 0.23$ & $0.57 \pm 0.24$ & $0.47 \pm 0.19$ & $0.68 \pm 0.22$ & $0.53 \pm 0.18$ \\
\hline GS decline (m/s observed) & $-0.15 \pm 0.20$ & & $-0.12 \pm 0.2$ & & $-0.15 \pm 0.20$ \\
\hline Baseline GS (m/s observed + imputed) & & $0.57 \pm 0.24$ & $0.36 \pm 0.25$ & $0.68 \pm 0.22$ & $0.51 \pm 0.20$ \\
\hline GS decline $(\mathrm{m} / \mathrm{s}$ observed + imputed $)$ & $-0.18 \pm 0.22$ & & $-0.21 \pm 0.25$ & & $-0.17 \pm 0.21$ \\
\hline Used walking aid in GS test, $n(\%)$ & $40(13.6)$ & $23(23.5)$ & $36(55.4)^{\mathrm{a}}$ & $17(8.6)$ & $55(27.8)^{\mathrm{b}}$ \\
\hline
\end{tabular}

Note. Data presented as mean \pm standard deviation or N (\%), unless otherwise stated. GS = gait speed; MMSE = Mini-Mental State Examination.

${ }^{\mathrm{a}} 33$ (33.7\%) missing GS data, and hence no data on walking aid use. ${ }^{\mathrm{b}} 19$ (9.6\%) missing GS data, and hence no data on walking aid use.

participants developed dementia after 5 years. The $\triangle$ MMSE and $\Delta \mathrm{GS}$ observed for each group are illustrated in Figures 2 and 3, respectively.

In logistic regression analysis, no association between $\Delta \mathrm{GS}$ and developing dementia was indicated prior to adjustment. In the adjusted analyses, $\Delta \mathrm{GS}$ was associated with developing dementia (Table 3). Baseline GS was associated with developing dementia in unadjusted and adjusted analyses (Table 3). In unadjusted and adjusted linear regression models, $\triangle \mathrm{GS}$ was significantly associated with $\triangle \mathrm{MMSE}$ in the total sample and in the Dem group, but not in the NoDem group (Table 4).

Although the omission of imputed GS values altered some results, the linear association between baseline GS and dementia was unchanged because there were no missing baseline GS values. The $\Delta \mathrm{GS}$ was not linearly associated with subsequent development of dementia in unadjusted $(\beta=0.45,95 \% \mathrm{CI}=[0.11,1.89], p=.273)$ or adjusted analyses $(\beta=0.85,95 \% \mathrm{CI}=[0.18,4.01], p=.838)$. In the total sample, $\Delta \mathrm{GS}$ did not associate significantly with $\triangle \mathrm{MMSE}$ in unadjusted or adjusted analyses $(\beta=0.30,95 \% \mathrm{CI}=[-4.02$, $3.42], p=.875$ and $\beta=1.21,95 \% \mathrm{CI}=[-2.61,5.04], p=.533$, respectively). In the Dem group, $\Delta \mathrm{GS}$ was significantly associated with $\triangle$ MMSE in adjusted analyses $(\beta=8.89,95 \% \mathrm{CI}=[0.09$, $17.69], p=.048)$, but not in unadjusted analyses $(\beta=6.10,95 \%$ $\mathrm{CI}=[-2.01,14.22], p=.138)$. In the NoDem group, $\Delta \mathrm{GS}$ and $\triangle \mathrm{MMSE}$ were not associated (unadjusted $\beta=-0.75,95 \% \mathrm{CI}=$ $[-3.26,1.75], p=.553$ and adjusted $\beta=-0.69,95 \% \mathrm{CI}=[-3.34,1.96]$, $p=.607)$.

\section{Discussion}

In this longitudinal study of very old people without dementia at baseline, including community and nursing home residents, we found that a decline in GS and a low baseline GS were independently associated with higher odds of dementia development within 5 years, after adjusting for potential confounders. In addition, declining GS was associated with declining cognitive function among those that developed dementia, but not among people that remained dementia-free.

The present results extend the findings of previous studies showing an association between declining GS and dementia development in studies involving primarily younger old people (Dumurgier et al., 2017; Montero-Odasso et al., 2018; Welmer et al., 2014). These studies, including ours, were thoroughly adjusted for potential confounders, such as age, sex, and morbidity.

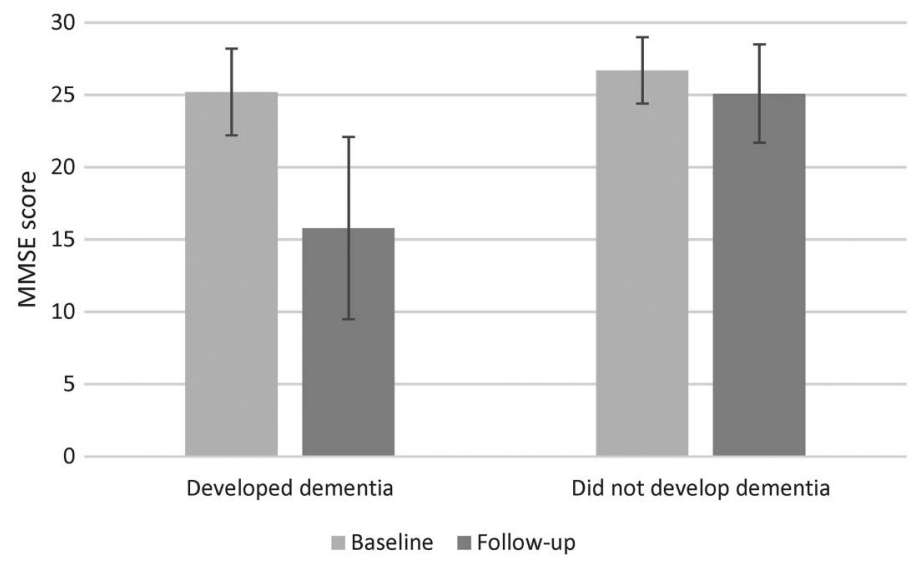

Figure 2 - MMSE scores at baseline and follow-up by dementia development group. Error bars represent $1 S D$. MMSE= Mini-Mental State Examination.

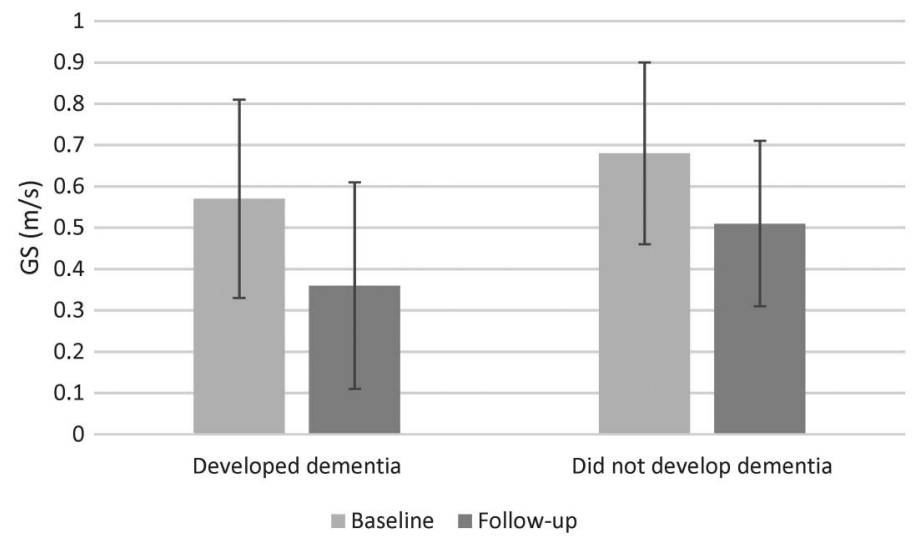

Figure 3 - GS (in meter per second) at baseline and follow-up by dementia development group. These data include imputed GS values at follow-up. Error bars represent $1 S D$. GS = gait speed.

It is especially important to adjust for potential confounders when investigating the association between gait and cognition in very old people due to the increasing prevalence of clinical diagnoses that may affect gait and/or cognition with increasing age. This 
Table 3 ORs for GS at Baseline and Change in GS for the Incidence of Dementia Within 5 Years

\begin{tabular}{lcccc}
\hline \multirow{2}{*}{ Measure } & Unadjusted & & Adjusted & \\
\cline { 2 - 4 } & OR $\mathbf{( 9 5 \% ~ C l )}$ & & OR (95\% Cl) & $\boldsymbol{p}$ \\
\hline$\Delta \mathrm{GS}^{\mathrm{a}}$ & $2.37[0.79,7.11]$ & .124 & $4.38[1.33,14.43]$ & .015 \\
GS at baseline $^{\mathrm{b}}$ & $8.04[2.51,25.77]$ & $<.001$ & $4.33[1.03,18.14]$ & .045 \\
\hline
\end{tabular}

Note. ADL = activities of daily living; BMI, body mass index; GS = gait speed; MMSE = Mini-Mental State Examination; ORs $=$ odds ratios; $\mathrm{CI}=$ confidence interval.

${ }^{a}$ Adjusted for age, sex, BMI, history of malignancy, nursing home resident, and Barthel ADL index score. ${ }^{\mathrm{b}}$ Adjusted for age, sex, BMI, history of malignancy, nursing home resident, Barthel ADL index score, history of hip fracture, and MMSE score.

Table 4 Linear Association Between Decline in GS and Decline in MMSE Scores in Total Sample and According to Dementia Development Within 5 Years

\begin{tabular}{lccccc}
\hline & Unadjusted & & \multicolumn{1}{c}{ Adjusted } & \\
\cline { 2 - 2 } Group & $\boldsymbol{\beta}(\mathbf{9 5 \%} \mathbf{~ C l})$ & & $\boldsymbol{\beta}$ & $\boldsymbol{\beta}$ (95\% Cl) & $\boldsymbol{p}$ \\
\hline Total $^{\mathrm{a}}$ & $6.41[3.40,9.41]$ & & $<.001$ & $7.17[4.17,10.18]$ & $<.001$ \\
Developed dementia $_{\text {Did not develop dementia }}$ & $11.74[7.01,16.39]$ & $<.001$ & & $13.01[8.32,17.83]$ & $<.001$ \\
\hline
\end{tabular}

Note. $\mathrm{BMI}=$ body mass index; $\mathrm{GS}=$ gait speed; MMSE = Mini-Mental State Examination; $\mathrm{CI}=$ confidence interval; $\beta=$ unstandardized beta.

${ }^{a}$ Adjusted for age, sex, BMI, history of malignancy, and use of walking aid during GS test.

circumstance may help to explain why we found an association between $\Delta \mathrm{GS}$ and dementia development only after adjusting for potential confounders. Another plausible explanation for the influence of potential confounders is the relatively low power of the analysis given the limited sample size retained throughout the study period.

We found that baseline GS was associated with increased odds of dementia development in very old people after adjusting for baseline cognitive performance. Although low baseline GS has been found to precede dementia in younger old people (Beauchet et al., 2016; Dumurgier et al., 2017), studies including only very old people are few. Notably, Bullain et al. (2016) found that baseline GS was associated with dementia development in people aged 90 years and over in analyses thoroughly adjusted for potential confounders, as was done here. The former also included baseline cognitive status determined by in-person neurological examination and cognitive measures of MMSE, the modified MMSE, or the Clinical Dementia Rating scale; and in this study, we adjusted for MMSE scores at baseline. Therefore, GS seems to be independently associated with dementia development and may provide unique and clinically useful information regarding risk of dementia that is not evident from direct assessments of cognitive function among very old people.

The association between decline in GS and dementia development was further strengthened by our observation that decline in GS was associated with decline in cognitive function in people that developed dementia. A recent meta-analysis showed that participants with declines in both GS and memory task performance were at higher risk for dementia development than those with decline in only one of these dimensions (Tian et al., 2020). Conversely, in a large study Hackett et al. (2018) reported no support for a synergistic effect between GS and cognition when cognition was assessed with an aggregate measure involving memory, time orientation, verbal fluency, and processing speed. The present lack of a significant association between declines in GS and cognitive function in our NoDem group is consistent with
Welmer et al.'s (2014) finding that cognitive function remained relatively stable while GS declined over 6 years in older people (mean age, 72 years) that did not develop dementia.

The fact that almost two thirds (33/51) of our participants with missing GS values at follow-up developed dementia may reflect age-related increases in both gait and cognitive deficits as well as the putative association between these two parameters. It is reasonable to infer that a weakening in statistical power due to the large proportion of missing GS values at follow-up explains, at least to some extent, why the association of GS with dementia development only became significant after we compensated for missing GS values. Multiple imputations were used to estimate missing GS values, with a preplanned strategy that adapted the imputations according to the reason for the missing value. Multiple imputation reduces the risk of Type 1 errors by building in variation (noise) through the creation of multiple data sets with statistically likely values for the entire sample based on available information for each individual (Dziura, Post, Zhao, Fu, \& Peduzzi, 2013).

A strength of this study is that it investigated the association between GS and cognition over 5 years among very old people. Another strength is that, we included participants with missing GS values, which should be expected to be prevalent in this age group, and used a preplanned strategy to impute missing GS values. Because comorbidities that can influence gait and cognition are common in the target age group of this study, our analyses were also thoroughly adjusted for potential confounders. Furthermore, performing the testing procedures in participants' homes enabled us to include those with mobility impairments and those who reside far from our institution. An experienced geriatric medicine specialist verified all medical diagnoses, thereby reducing the risk of dementia misclassification. The study's weaknesses entail the selectivity of our inclusion criteria, which required being 85 years or older and then surviving another 5 years. Consequently, our sample was likely biased to healthier very old people, somewhat limiting the generalizability of our findings. We were able to obtain a 5-year follow-up data for 296 individuals without 
dementia at baseline, of almost 2,000 persons aged 85 years and over. This final sample size, with 98 all-cause dementia cases over 5 years, limited further subgroup analyses of potential relationships of GS with particular types of dementia. Finally, although it is widely used, because the MMSE is a screening instrument, it only estimates global cognitive function without targeted assessments of frontal lobe function or processing speed, which are often affected by dementia.

In practice, GS tests seem to be able to screen for mortality risk and for declining physical and cognitive health in older people, including very old people (Abellan Van Kan et al., 2009; Cummings, Studenski, \& Ferrucci, 2014; Dumurgier et al., 2017; Toots et al., 2013), and can complement cognitive tests in the prediction of dementia. Our observation of declining GS with age, with or without development of dementia suggests that albeit unspecific, a decline in GS can reveal the need for further investigation. Screening for low GS and GS decline may identify persons at elevated risk of dementia, such that they can then be monitored more closely over time. The GS of both the Dem and NoDem group declined over 5 years, at 0.21 and $0.17 \mathrm{~m} / \mathrm{s}$, respectively. In comparison, substantial meaningful change has been reported to be between 0.08 to $0.11 \mathrm{~m} / \mathrm{s}$ in communitydwelling older people (Perera, Mody, Woodman, \& Studenski, 2006) and minimal clinically important difference between 0.10 and $0.20 \mathrm{~m} / \mathrm{s}$ in community-dwelling older people and in multiple patient groups of older people (Bohannon \& Glenney, 2014). A GS threshold of $>1.0 \mathrm{~m} /$ s has been suggested (Dumurgier et al., 2017), but that cutoff may be too fast for very old people given that few in our sample walked faster than $1 \mathrm{~m} / \mathrm{s}$. Still, there remains a need to establish suitable GS thresholds to indicate risk of dementia among very old people.

\section{Conclusion}

Our study showed that a low or declining GS was associated with higher odds of dementia development in people aged 85 and over, including both community and nursing home residents. Furthermore, declining GS was associated with declining cognitive function among those who developed dementia. The present results support the development of a GS screening index for predicting future cognitive decline risk, even among very old people. Optimal GS cutoff scores and combination with cognitive parameters to best predict dementia development is an interesting field of future research.

\section{References}

Abellan Van Kan, G.Y., Rolland, S.A., Bauer, J., Beauchet O., Bonnefoy, M., Cesari, M., ... Vellas, B. (2009). Gait speed at usual pace as a predictor of adverse outcomes in community-dwelling older people an international academy on nutrition and aging (IANA) task force. The Journal of Nutrition, Health \& Aging, 13(10), 881-889. doi:10. 1007/s12603-009-0246-Z

American Psychiatric Association [APA]. (2000). Diagnostic and statistical manual of mental disorders. IV-Text Revision. Washington, DC: Author.

Beauchet, O., Annweiler, C., Callisaya, M.L., De Cock, A.-M., Helbostad, J.L., Kressig, R.W., . . . A Allali, G. (2016). Poor gait performance and prediction of dementia: Results from a meta-analysis. Journal of the American Medical Directors Association, 17(6), 482-490. PubMed ID: 26852960 doi:10.1016/j.jamda.2015.12.092

Bohannon, R.W., \& Glenney, S.S. (2014). Minimal clinically important difference for change in comfortable gait speed of adults with pathology: A systematic review. Journal of Evaluation in Clinical
Practice, 20(4), 295-300. PubMed ID: 24798823 doi:10.1111/jep. 12158

Bullain, S.S., Corrada, M.M., Perry, S.M., \& Kawas, C.H. (2016). Sound body sound mind? Physical performance and the risk of dementia in the oldest-old: The 90+ study. Journal of the American Geriatrics Society, 64(7), 1408-1415. PubMed ID: 27377238 doi:10.1111/jgs. 14224

Calderón-Larrañaga, A., Vetrano, D.L., Ferrucci, L., Mercer, S.W., Marengoni, A., Onder, G., .. . Fratiglioni, L. (2019). Multimorbidity and functional impairment-bidirectional interplay, synergistic effects and common pathways. Journal of Internal Medicine, 285(3), 255271. PubMed ID: 30357990 doi:10.1111/joim.12843

Collin, C., Wade, D.T., Davies, S., \& Horne, V. (1988). The Barthel ADL index: A reliability study. International Disability Studies, 10(2), 61-63. PubMed ID: 3403500 doi:10.3109/09638288809164103

Creavin, S.T., Wisniewski, S., Noel-Storr, A.H., Trevelyan, C.M., Hampton, T., Rayment, D., ... Cullum, S. (2016). Mini-Mental State Examination (MMSE) for the detection of dementia in clinically unevaluated people aged 65 and over in community and primary care populations. The Cochrane Database of Systematic Reviews, (1), CD011145. doi:10.1002/14651858.cd011145.pub2

Cummings, S.R., Studenski, S., \& Ferrucci, L. (2014). A diagnosis of dismobility-Giving mobility clinical visibility. Journal of the American Medical Association, 311(20), 2061-2062. PubMed ID: 24763978 doi:10.1001/jama.2014.3033

Dumurgier, J., Artaud, F., Touraine, C., Rouaud, O., Tavernier, B., Dufouil, C., ... Elbaz, A. (2017). Gait speed and decline in gait speed as predictors of incident dementia. The Journals of Gerontology. Series A, Biological Sciences and Medical Sciences, 72(5), 655-61. PubMed ID: 27302701 doi:10.1093/gerona/glw110

Dziura, J.D., Post, L.A., Zhao, Q., Fu, Z., \& Peduzzi, P. (2013). Strategies for dealing with missing data in clinical trials: From design to analysis. The Yale Journal of Biology and Medicine, 86(3), 343-58. PubMed ID: 24058309

Folstein, M.F., Folstein, S.E., and McHugh, P.R. (1975). Mini-mental state. Journal of Psychiatric Research, 12(3), 189-198. PubMed ID: 1202204 doi:10.1016/0022-3956(75)90026-6

Grande, G., Triolo, F., Nuara, A., Welmer, A.K., Fratiglioni, L., \& Vetrano, D.L. (2019). Measuring gait speed to better identify prodromal dementia. Experimental Gerontology, 124, 110625. PubMed ID: 31173841 doi:10.1016/j.exger.2019.05.014

Guralnik, J.M., Simonsick, E.M., Ferrucci, L., Glynn, R.J., Berkman, L.F., Blazer, D.G., ... Wallace, R.B. (1994). A short physical performance battery assessing lower extremity function: Association with self-reported disability and prediction of mortality and nursing home admission. Journal of Gerontology, 49(2), M85-M94. PubMed ID: 8126356 doi:10.1093/geronj/49.2.M85

Hackett, R.A., Davies-Kershaw, H., Cadar, D., Orrell, M., \& Steptoe, A. (2018). Walking speed, cognitive function, and dementia risk in the English longitudinal study of ageing. Journal of the American Geriatrics Society, 66(9), 1670-1675. PubMed ID: 29508385 doi:10.1111/jgs.15312

Hayati Rezvan, P., Lee, K.J., \& Simpson, J.A. (2015). The rise of multiple imputation: A review of the reporting and implementation of the method in medical research. BMC Medical Research Methodology, 15(1), 30. doi:10.1186/s12874-015-0022-1

Jensen, E., Dehlin, O., \& Gustafson, L. (1993). A comparison between three psychogeriatric rating scales. International Journal of Geriatric Psychiatry, 8(3), 215-229. doi:10.1002/gps.930080305

Lawton, M.P. (1975). The Philadelphia geriatric center morale scale: A revision. Journal of Gerontology, 30(1), 85-89. PubMed ID: 1109399 doi:10.1093/geronj/30.1.85 
Livingston, G., Sommerlad, A., Orgeta, V., Costafreda, S.G., Huntley, J., Ames, D., ... Mukadam, N. (2017). Dementia prevention, intervention, and care. The Lancet, 390(10113), 2673-2734. doi:10.1016/ S0140-6736(17)31363-6

Middleton, A., Fritz, S.L., \& Lusardi, M. (2015). Walking speed: The functional vital sign. Journal of Aging and Physical Activity, 23(2), 314-322. PubMed ID: 24812254 doi:10.1123/japa.2013-0236

Montero-Odasso, M., Speechley, M., Muir-Hunter, S.W., SarquisAdamson, Y., Sposato, L.A., Hachinski, V., ... \& The Canadian Gait and Cognition Network. (2018). Motor and cognitive trajectories before dementia: Results from gait and brain study. Journal of the American Geriatrics Society, 66(9), 1676-1683. doi:10.1111/jgs. 15341

Morris, R., Lord, S., Bunce, J., Burn, D., \& Rochester, L. (2016). Gait and cognition: Mapping the global and discrete relationships in ageing and neurodegenerative disease. Neuroscience and Biobehavioral Reviews, 64, 326-345. PubMed ID: 26915926 doi:10.1016/j. neubiorev.2016.02.012

Niklasson, J., Conradsson, M., Hörnsten, C., Nyqvist, F., Padyab, M., Nygren B., ... Gustafson, Y. (2015). Psychometric properties and feasibility of the Swedish version of the Philadelphia geriatric center morale scale. Quality of Life Research: An International Journal of Quality of Life Aspects of Treatment, Care and Rehabilitation, 24(11), 2795-2805. doi:10.1007/s11136-015-1009-4

Öhlin, J., Ahlgren, A., Folkesson, R., Gustafson, Y., Littbrand, H., Olofsson, B., \& Toots, A. (2020). The association between cognition and gait in a representative sample of very old people-The influence of dementia and walking aid use. BMC Geriatrics, 20(1), 34. PubMed ID: 32005103 doi:10.1186/s12877-020-1433-3

Peel, N.M., Kuys, S.S., \& Klein, K. (2013). Gait speed as a measure in geriatric assessment in clinical settings: A systematic review. The Journals of Gerontology. Series A, Biological Sciences and Medical Sciences, 68(1), 39-46. PubMed ID: 22923430 doi:10.1093/gerona/ gls174

Perera, S., Mody, S.H., Woodman, R.C., \& Studenski, S.A. (2006). Meaningful change and responsiveness in common physical performance measures in older adults. Journal of the American Geriatrics Society, 54(5), 743-749. PubMed ID: 16696738 doi:10.1111/j.15325415.2006.00701.x
Sheikh, J.I.\& Yesavage, J.A. (1986). Geriatric Depression Scale (GDS): Recent evidence and development of a shorter version. Clinical Gerontologist, 5(1-2), 165-173. doi:10.1300/j018v05n01_09

Studenski, S., Perera, S., Patel, K., Rosano, C., Faulkner, K., Inzitari, M., ... Guralnik, J. (2011). Gait speed and survival in older adults. Journal of the American Medical Association, 305(1), 50-58. PubMed ID: 21205966 doi:10.1001/jama.2010.1923

Tian, Q., Resnick, S.M., Mielke, M.M., Yaffe, K., Launer, L.J., Jonsson, P.V., ... Ferrucci, L. (2020). Association of dual decline in memory and gait speed with risk for dementia among adults older than 60 years: A multicohort individual-level meta-analysis. JAMA Network Open, 3(2), e1921636-e1921636. PubMed ID: 32083691 doi:10.1001/ jamanetworkopen.2019.21636

Tilvis, R.S., Laitala, V., Routasalo, P., Strandberg, T.E., \& Pitkala, K.H. (2012). Positive life orientation predicts good survival prognosis in old age. Archives of Gerontology and Geriatrics, 55(1), 133-137. PubMed ID: 21764146 doi:10.1016/j.archger.2011. 06.030

Tombaugh, T.N.\& McIntyre, N.J. (1992). The Mini-Mental State Examination: A comprehensive review. Journal of the American Geriatrics Society, 40(9), 922-935. PubMed ID: 1512391 doi:10.1111/j.15325415.1992.tb01992.x

Toots, A., Rosendahl, E, Lundin-Olsson, L., Nordström, P., Gustafson, Y., \& Littbrand, H. (2013). Usual gait speed independently predicts mortality in very old people: A population-based study. Journal of the American Medical Directors Association, 14(7), 529.e1-529.e6. doi:10.1016/j.jamda.2013.04.006

United Nations, Department of Economic and Social Affairs, Population Division [UN/DESA]. (2015). "World Population Ageing."

van Buren, S. (2012). Flexible imputation of missing data (1st ed.). Boca Raton, FL: Taylor and Francis Group. doi: 10.1201/b11826

Welmer, A.-K., Rizzuto, D., Qiu, C., Caracciolo, B., \& Laukka, E.J. (2014). Walking speed, processing speed, and dementia: A population-based longitudinal study. The Journals of Gerontology. Series A, Biological Sciences and Medical Sciences, 69(12), 1503-1510. PubMed ID: 24706441 doi:10.1093/gerona/glu047

World Health Organization, Alzheimer Disease International [WHO/ADI]. (2012). Dementia: A public health priority. Geneva, Switzerland: Author. 